\title{
Conservation Laws and Exact Solutions for a Reaction-Diffusion Equation with a Variable Coefficient
}

\author{
Zhijie Cao ${ }^{1,2}$ and Yiping Lin ${ }^{1}$ \\ ${ }^{1}$ Department of Applied Mathematics, Kunming University of Science and Technology, Kunming, Yunnan 650093, China \\ ${ }^{2}$ Department of Mathematics, College of Science, China Three Gorges University, Yichang, Hubei 443002, China \\ Correspondence should be addressed to Zhijie Cao; czj0092003@aliyun.com
}

Received 5 June 2014; Accepted 19 July 2014; Published 5 August 2014

Academic Editor: Linghai Zhang

Copyright (c) 2014 Z. Cao and Y. Lin. This is an open access article distributed under the Creative Commons Attribution License, which permits unrestricted use, distribution, and reproduction in any medium, provided the original work is properly cited.

In this paper a variable-coefficient reaction-diffusion equation is studied. We classify the equation into three kinds by different restraints imposed on the variable coefficient $b(x)$ in the process of solving the determining equations of Lie groups. Then, for each kind, the conservation laws corresponding to the symmetries obtained are considered. Finally, some exact solutions are constructed.

\section{Introduction}

Conservation law is an important concept in physics. It describes a quantity that is conserved; that is, the total amount is the same before and after something occurs. In mathematics, conservation law provides one of the basic principles in formulating and investigating models. For instance, sometimes, the existence of a large amount of conservation laws of a partial differential equation (PDE) is a strong indication of its integrability.

For an Euler-Lagrange equation, which arises out of a variational principle, the remarkable Noether's theorem [1] constructed a one-to-one correspondence between a nontrivial generalized variational symmetry of some functionals and a nontrivial conservation law. By this correspondence, one can establish conservation laws for an Euler-Lagrange equation, as illustrated in [2], whereas for a system not arising from a variational principle, such as a single evolution equation, researchers have made various generalizations [313] of Noether's method to construct conservation laws.

Among these generalizations, after suggesting some concepts, such as adjoint equation, strict self-adjointness, quasiself-adjointness, and nonlinear self-adjointness, Ibragimov [3] derived a convenient formula to establish conservation laws for the simultaneous system of the target equation together with its adjoint equation. By this formula, one can construct a conservation law for the combined system through a formal Lagrangian $\mathscr{L}$ corresponding to any Lie point, Lie-Bäcklund, or nonlocal symmetry. But $\mathscr{L}$ involves a "nonphysical" variable $v$. If the equation considered is (nonlinearly) self-adjoint, one can eliminate $v$ via a certain substitution to obtain the conservation law of the original equation. However, if the equation does not have any selfadjoint property, the conservation laws for it corresponding to each symmetry can be considered as local conservation laws of the simultaneous system but reflect the symmetry property of the original equation.

Following Ibragimov, many researchers have been studying this interesting area and there are a lot of works in the literature, such as [14-19] and the references therein.

In this paper, we consider a variable-coefficient reactiondiffusion equation [20]

$$
E \equiv u_{t}-d u_{x x}-b(x) u+u^{2}=0
$$

where $u=u(x, t)$ represents the population density, $d(\neq 0)$ is a constant of the diffusion rate, and the variable-coefficient $b(x)$ describes the growth rate of the living being. As a diffusive logistic equation, (1) describes the dynamics of a population inhabiting a strongly hetergeneous environment. The growth rate $b(x)$ is positive on favourable habitats and negative on unfavorable ones. In [20], Cantrell and Cosner determined how the spatial arrangement of favourable and unfavourable habitats affects the population being modelled. 
In particular, $b(x) \equiv$ const. $=b$ makes $(1)$ in the form

$$
u_{t}=d u_{x x}+R(u)
$$

where $R(u)=b u-u^{2}$, which is the nonlinear heat equation [21] when $d=1$.

In [22], Ide and Okada designed numerical schemes that preserve energy property and showed numerical experiments for (1).

We start our work with solving the system of determining equations to obtain the infinitesimal generator and meantime classifying (1) into three kinds. To determine the form of the conservation law corresponding to any symmetry of every kind, we then study the self-adjointness of (1). By the theorem [23] on the order of conservation laws for a general class of second-order evolution equations, which covers (1), we illustrate that the local conservation law of (1) does not exist. With the aid of Ibragimov's formula, we construct conservation laws corresponding to every symmetry of each kind. Finally, using the symmetry group obtained and another method, we establish some exact solutions of the last two kinds of (1).

The contents of this paper are as follows. In Section 2, we will give some preliminaries, namely, some definitions, Ibragimov's theorem, and a theorem on the order of local conservation laws for a general class of second-order evolution equations which covers (1). Next we subject (1) to the effective computational procedure [2] for finding the Lie point symmetries and study its self-adjointness in Section 3. Section 4 is devoted to discussing the conservation laws of (1). Some exact solutions of (1) are obtained in Section 5. Finally, concluding remarks are given in Section 6.

\section{Preliminaries}

We assume that all functions are smooth, and the summation over the repeated indices is understood.

2.1. Ibragimov's Theory. In this subsection, we recall Ibragimov's procedure of constructing conservation laws corresponding to the given symmetries of any system of PDEs, provided that the number of equations in the system is equal to the number of dependent variables.

For convenience, we consider a scalar evolution equation

$$
F\left(x, u, u_{(1)}, \ldots, u_{(s)}\right)=0
$$

with independent variables $x=\left(x^{1}, x^{2}\right)$ (here denote $x^{1}=$ $x, x^{2}=t$.) and a dependent variable $u$, where $u_{(1)}=$ $\left\{u_{i}\right\}, u_{(2)}=\left\{u_{i j}\right\}, \ldots$ denote the sets of the partial derivatives of the first, second, and so forth, orders, $u_{i}=\partial u / \partial x^{i}, u_{i j}=$ $\partial^{2} u / \partial x^{i} \partial x^{j}, \ldots$

Definition 1. The adjoint equation to (3) is

$$
F^{*}\left(x, u, v, u_{(1)}, v_{(1)}, \ldots, u_{(s)}, v_{(s)}\right)=0,
$$

with

$$
F^{*}\left(x, u, v, u_{(1)}, v_{(1)}, \ldots, u_{(s)}, v_{(s)}\right)=\frac{\delta(v F)}{\delta u}
$$

where $v=v\left(x^{1}, x^{2}\right)$ is a multiplier and

$$
\frac{\delta}{\delta u}=\frac{\partial}{\partial u}+\sum_{s=1}^{\infty}(-1)^{s} D_{i_{1}} \cdots D_{i_{s}} \frac{\partial}{\partial u_{i_{1} \cdots i_{s}}}
$$

denotes the variational derivatives (the Euler-Lagrange operator) and

$$
D_{i}=\frac{\partial}{\partial x^{i}}+u_{i} \frac{\partial}{\partial u}+u_{i j} \frac{\partial}{\partial u_{j}}+\cdots
$$

are the total differentiations.

We now extend (3) to a system

$$
\begin{gathered}
\frac{\delta(v F)}{\delta v}=F\left(x, u, u_{(1)}, \ldots, u_{(s)}\right)=0 \\
\frac{\delta(v F)}{\delta u}=F^{*}\left(x, u, v, u_{(1)}, v_{(1)}, \ldots, u_{(s)}, v_{(s)}\right)=0 .
\end{gathered}
$$

In [3], Ibragimov proved that (8) inherits all symmetries of (3) and, using Noether's identity [13], obtained a formula of conservation law corresponding to every symmetry of (3).

Theorem 2. Any Lie point, Lie-Bäcklund, or nonlocal symmetry

$$
V=\xi^{i}\left(x, u, u_{(1)}, \ldots\right) \frac{\partial}{\partial x^{i}}+\eta\left(x, u, u_{(1)}, \ldots\right) \frac{\partial}{\partial u}
$$

of (3) provides a conservation law $D_{i}\left(C^{i}\right)=0$ for $(8)$. The conserved vector is given by

$$
\begin{aligned}
C^{i}= & \xi^{i} \mathscr{L} \\
& +w\left[\frac{\partial \mathscr{L}}{\partial u_{i}}-D_{j}\left(\frac{\partial \mathscr{L}}{\partial u_{i j}}\right)+D_{j} D_{k}\left(\frac{\partial \mathscr{L}}{\partial u_{i j k}}\right)-\cdots\right] \\
& +D_{j}(w)\left[\frac{\partial \mathscr{L}}{\partial u_{i j}}-D_{k}\left(\frac{\partial \mathscr{L}}{\partial u_{i j k}}\right)+D_{k} D_{r}\left(\frac{\partial \mathscr{L}}{\partial u_{i j k r}}\right)-\cdots\right] \\
& +D_{j} D_{k}(w)\left[\frac{\partial \mathscr{L}}{\partial u_{i j k}}-D_{r}\left(\frac{\partial \mathscr{L}}{\partial u_{i j k r}}\right)+\cdots\right]+\cdots,
\end{aligned}
$$

where $w$ and $\mathscr{L}$ are defined as follows:

$$
w=\eta-\xi^{j} u_{j}, \quad \mathscr{L}=v F\left(x, u, u_{(1)}, \ldots, u_{(s)}\right) .
$$

For a second-order equation, (10) becomes

$$
C^{i}=\xi^{i} \mathscr{L}+w\left[\frac{\partial \mathscr{L}}{\partial u_{i}}-D_{j}\left(\frac{\partial \mathscr{L}}{\partial u_{i j}}\right)\right]+D_{j}(w) \frac{\partial \mathscr{L}}{\partial u_{i j}} .
$$

According to Theorem 2, we know that every symmetry of (3) can provide a conservation law. To express these conservation laws one depends on not only the original variables but the multiplier $v$.

Sometimes the multiplier $v$ can be removed from the conservation law provided that (3) has a property of selfadjointness. 
Definition 3. Equation (3) is said to be self-adjoint if the equation obtained from the adjoint equation (4) by the substitutions $v=u$

$$
F^{*}\left(x, u, v, u_{(1)}, v_{(1)}, \ldots, u_{(s)}, v_{(s)}\right)=0
$$

is identical with the original equation (3), in other words, if

$$
\begin{aligned}
F^{*} & \left.\left(x, u, v, u_{(1)}, v_{(1)}, \ldots, u_{(s)}, v_{(s)}\right)\right|_{v=u} \\
& =\phi\left(x, u, u_{(1)}, \ldots\right) F\left(x, u, u_{(1)}, \ldots, u_{(s)}\right)
\end{aligned}
$$

Definition 4. Equation (3) is said to be quasi-self-adjoint if the equation obtained from the adjoint equation (4) by the substitutions $v=\varphi(u)$ with a certain function $\varphi(u)$ such that $\varphi^{\prime}(u) \neq 0$

$$
F^{*}\left(x, u, v, u_{(1)}, v_{(1)}, \ldots, u_{(s)}, v_{(s)}\right)=0
$$

is identical with the original equation (3).

Definition 5. Equation (3) is said to be weak self-adjoint if the equation obtained from the adjoint equation (4) by the substitutions $v=\varphi(x, u)$ with a certain function $\varphi(x, u)$ such that $\varphi_{u} \neq 0$ and $\varphi_{x} \neq 0$

$$
F^{*}\left(x, u, v, u_{(1)}, v_{(1)}, \ldots, u_{(s)}, v_{(s)}\right)=0
$$

is identical with the original equation (3).

Definition 6. Equation (3) is nonlinearly self-adjoint if there exist functions $v=\varphi(x, u)$ that solve the adjoint equation (4) for all solutions $u(x)$ of (3) and satisfy the condition $\varphi(x, u) \neq$ 0 .

Remark 7. The first three definitions were suggested by Ibragimov in $[3,9,24]$, respectively, the fourth was introduced by Gandarias in [25], and few time later it was generalized by Ibragimov in [13] in the form of Definition 6 . So the nonlinear self-adjointness can be seen the most general concept; namely, the others are all its trivial cases.

2.2. A Theorem on the Order of Local Conservation Laws for a General Class of Second-Order Evolution Equations. The theorem on the order of local conservation laws for a more general class of second-order evolution equations, which covers (1), has been proved by Popovych in [23].

Theorem 8. Any local conservation law of any second-order $(1+1)$-dimensional quasilinear evolution equation, whose form is

$$
u_{t}=S\left(t, x, u, u_{x}\right) u_{x x}+R\left(t, x, u, u_{x}\right)
$$

where $S\left(t, x, u, u_{x}\right) \neq 0$, has the first order and, moreover, there exists its conserved vector with the density $T$ depending at most on $t, x$, and $u$ and the flux $X$ depending at most on $t, x, u$, and $u_{x}$.

\section{Lie Point Symmetries and Self-Adjointness}

In this section, we present the most general Lie group of point transformations, which leaves (1) invariant, and study the self-adjointness of (1).

First of all, we consider a one-parameter Lie group of infinitesimal transformation:

$$
\begin{gathered}
x \longrightarrow x+\epsilon \xi(x, t, u), \\
t \longrightarrow t+\epsilon \tau(x, t, u), \\
u \longrightarrow u+\epsilon \phi(x, t, u),
\end{gathered}
$$

with a small parameter $\epsilon \ll 1$. The vector field associated with the above group of transformations can be written as

$$
V=\xi(x, t, u) \frac{\partial}{\partial x}+\tau(x, t, u) \frac{\partial}{\partial t}+\phi(x, t, u) \frac{\partial}{\partial u} .
$$

Applying the second prolongation

$$
\begin{aligned}
\operatorname{pr}^{(2)} V= & V+\phi^{x} \frac{\partial}{\partial u_{x}}+\phi^{t} \frac{\partial}{\partial u_{t}} \\
& +\phi^{x x} \frac{\partial}{\partial u_{x x}}+\phi^{x t} \frac{\partial}{\partial u_{x t}}+\phi^{t t} \frac{\partial}{\partial u_{t t}}
\end{aligned}
$$

to $(1)$, we find that the coefficient functions $\xi(x, t, u), \tau(x, t, u)$, and $\phi(x, t, u)$ must satisfy the symmetry condition

$$
\begin{aligned}
\phi^{t}-d \phi^{x x}-b^{\prime}(x) u \xi(x, t, u) & \\
& -b(x) \phi(x, t, u)+2 u \phi(x, t, u)=0,
\end{aligned}
$$

where $\phi^{t}, \phi^{x x}$ are the coefficients in $\operatorname{pr}^{(2)} V$ and

$$
\begin{aligned}
\phi^{t}= & D_{t} \phi(x, t, u)-u_{x} D_{t} \xi(x, t, u)-u_{t} D_{t} \tau(x, t, u), \\
\phi^{x x}= & D_{x}^{2} \phi(x, t, u)-u_{x} D_{x}^{2} \xi(x, t, u)-u_{t} D_{x}^{2} \tau(x, t, u) \\
& -2 u_{x x} D_{x} \xi(x, t, u)-2 u_{x t} D_{x} \tau(x, t, u),
\end{aligned}
$$

where $D_{x}, D_{t}$ are the total derivatives with respect to $x$ and $t$, respectively.

Substituting $\phi^{t}, \phi^{x x}$ into the symmetry condition, replacing $u_{t}$ by $d u_{x x}+b(x) u-u^{2}$ whenever it occurs, and equating the coefficients of the various monomials in the first- and second-order partial derivatives of $u$, we obtain the following system of determining equations:

$$
\begin{gathered}
\xi_{u}=0, \\
\tau_{u}=0, \\
\phi_{u u}=0, \\
\tau_{x}=0, \\
-d \tau_{t}+2 d \xi_{x}=0, \\
-\xi_{t}-2 d \phi_{x u}+d \xi_{x x}=0, \\
(-b(x)+2 u) \phi+u \xi b^{\prime}(x)+\left(-u^{2}+u b(x)\right) \phi_{u} \\
+\phi_{t}+\left(u^{2}-u b(x)\right) \tau_{t}-d \phi_{x x}=0 .
\end{gathered}
$$


The solution of the Subsystem (23)-(27) can be easily found to be

$$
\begin{gathered}
\tau(x, t, u)=\tau(t), \\
\phi(x, t, u)=\beta(x, t) u+\alpha(x, t), \\
\xi(x, t, u)=\frac{1}{2} \tau^{\prime}(t) x+p(t),
\end{gathered}
$$

for certain functions $\alpha, \beta$, and $p$.

By substituting $\tau(x, t, u), \phi(x, t, u)$, and $\xi(x, t, u)$ obtained in (28) and (29), respectively, we obtain

$$
\begin{gathered}
-\xi_{t}(x, t)-2 d \beta_{x}(x, t)=0 \\
-b(x)(\beta(x, t) u+\alpha(x, t)) \\
-b^{\prime}(x) u\left(\frac{1}{2} \tau^{\prime}(t) x+p(t)\right)+u b(x) \beta(x, t) \\
-u b(x) \tau^{\prime}(t)+u^{2}\left(\beta(x, t)+\tau^{\prime}(t)\right) \\
+u\left(2 \alpha(x, t)+\beta_{t}(x, t)-d \beta_{x x}(x, t)\right) \\
+\alpha_{t}(x, t)-d \alpha_{x x}(x, t)=0 .
\end{gathered}
$$

As $b(x)$ is uncertain, we split (34) into two parts: the part contains $b(x)$ and $b^{\prime}(x)$ and the remainder; that is,

$$
\begin{aligned}
& -b(x) \alpha(x, t)-b^{\prime}(x) u\left(\frac{1}{2} \tau^{\prime}(t) x+p(t)\right) \\
& \quad-u b(x) \tau^{\prime}(t)=0, \\
& u^{2}\left(\beta(x, t)+\tau^{\prime}(t)\right) \\
& +u\left(2 \alpha(x, t)+\beta_{t}(x, t)-d \beta_{x x}(x, t)\right) \\
& +\alpha_{t}(x, t)-d \alpha_{x x}(x, t)=0 .
\end{aligned}
$$

We rearrange (35) as

$$
\begin{aligned}
& u\left[-b(x) \tau^{\prime}(t)-b^{\prime}(x)\left(\frac{1}{2} \tau^{\prime}(t) x+p(t)\right)\right]-b(x) \alpha(x, t) \\
& \quad=0
\end{aligned}
$$

Then it splits into

$$
\begin{gathered}
-b(x) \tau^{\prime}(t)-b^{\prime}(x)\left(\frac{1}{2} \tau^{\prime}(t) x+p(t)\right)=0, \\
-b(x) \alpha(x, t)=0,
\end{gathered}
$$

Equation (36) splits into

$$
\begin{gathered}
\beta(x, t)+\tau^{\prime}(t)=0, \\
\alpha_{t}(x, t)-d \alpha_{x x}(x, t)=0, \\
2 \alpha(x, t)+\beta_{t}(x, t)-d \beta_{x x}(x, t)=0 .
\end{gathered}
$$

We obtain a simultaneous system Equation (33) and Equations (38)-(41). Equation (39) yields $\beta(x, t)=-\tau^{\prime}(t)$; then $\beta_{x}(x, t)=\beta_{x x}(x, t)=0$. Equation (41) yields $\alpha_{x}(x, t)=$ $\alpha_{x x}(x, t)=0$. Then, by (40), $\alpha_{t}(x, t)=0$, that is, $\alpha(x, t)$ is a constant. So (33) reduces to the form

$$
-\frac{1}{2} \tau^{\prime \prime}(t) x-p^{\prime}(t)=0,
$$

whence $\tau^{\prime \prime}(t)=0, p^{\prime}(t)=0$, and hence

$$
\tau(t)=c_{1} t+c_{2}, \quad p(t)=c_{3},
$$

where $c_{i}(i=1,2,3)$ are arbitrary constants. Ultimately, we arrive at $\alpha(x, t)=0$ and the general solution of the system of determining equations:

$$
\begin{gathered}
\tau(x, t, u)=c_{1} t+c_{2}, \\
\xi(x, t, u)=\frac{1}{2} c_{1} x+c_{3}, \\
\phi(x, t, u)=-c_{1} u .
\end{gathered}
$$

And, invoking (35), the restriction on the variable-coefficient $b(x)$ is

$$
\left(\frac{1}{2} c_{1} x+c_{3}\right) b^{\prime}(x)+c_{1} b(x)=0 .
$$

According to $c_{i}$, we can classify (1) into the following three kinds.

Case 1. When $c_{2}=1, c_{1}=c_{3}=0, b(x)$ is free and (1) has the infinitesimal generator

$$
V_{1}=\partial_{t}
$$

For (1) does not have any dependence on $t$, this is clear.

Case 2. When $c_{1}=1, c_{2}=c_{3}=0$, the restriction on $b(x)$ is $(1 / 2) x b^{\prime}(x)+b(x)=0$; namely, $b(x)=c_{4} / x^{2}$, where $c_{4}$ is an integral constant, and the infinitesimal generator is

$$
V_{2}=\frac{1}{2} x \partial_{x}+t \partial_{t}-u \partial_{u}
$$

where $\partial_{t}, \partial_{x}, \partial_{u}$ denote $\partial / \partial t, \partial / \partial x, \partial / \partial u$, respectively. So we conclude that the partial differential equation

$$
u_{t}-d u_{x x}-\frac{c_{4}}{x^{2}} u+u^{2}=0
$$

has the infinitesimal generators $V_{1}, V_{2}$. The commutation table of the Lie algebra generated by the infinite symmetries is

$$
\begin{array}{ccc}
{[\cdot, \cdot]} & V_{1} & V_{2} \\
V_{1} & 0 & V_{1} \\
V_{2} & -V_{1} & 0 .
\end{array}
$$

Case 3. When $c_{3}=1, c_{1}=c_{2}=0$, the restriction on $b(x)$ is $b^{\prime}(x)=0$; namely, $b(x)=$ const. $=b$, and the infinitesimal generator is

$$
V_{3}=\partial_{x}
$$


So we conclude that the partial differential equation

$$
u_{t}-d u_{x x}-b u+u^{2}=0
$$

has the infinitesimal generators $V_{1}, V_{3}$. The commutation table of the Lie algebra generated by the infinite symmetries is

$$
\begin{array}{ccc}
{[\cdot, \cdot]} & V_{1} & V_{3} \\
V_{1} & 0 & 0 \\
V_{3} & 0 & 0 .
\end{array}
$$

Now we study the self-adjoint property of (1).

The adjoint equation to (1) is

$$
\begin{aligned}
E^{*} & =\frac{\delta}{\delta u}[v(x, t) E] \\
& =-v b(x)+2 v u-v_{t}-d v_{x x}=0 .
\end{aligned}
$$

It is manifest that for $d \neq 0,(1)$ is not strictly self-adjoint.

Setting $v=h(u)$ in (53), we have

$$
\begin{aligned}
\left.E^{*}\right|_{v=h(u)}= & -h(u) b(x)+2 u h(u) \\
& -h^{\prime}(u) u_{t}-d h^{\prime \prime}(u) u_{x}^{2}-d h^{\prime}(u) u_{x x} .
\end{aligned}
$$

Equation (14) yields

$$
\begin{aligned}
-\left.E^{*}\right|_{v=h(u)}+\lambda E= & u_{t}\left(\lambda+h^{\prime}(u)\right)+d u_{x x}\left(-\lambda+h^{\prime}(u)\right) \\
& -\lambda u b(x)+\lambda u^{2}+d h^{\prime \prime}(u) u_{x}^{2} \\
& +h(u) b(x)-2 u h(u)=0 .
\end{aligned}
$$

Noting the coefficients of $u_{t}$ and $u_{x x}$, we obtain that $\lambda+h^{\prime}(u)=$ 0 and $-\lambda+h^{\prime}(u)=0$; that is $\lambda=h^{\prime}(u)=0$; this means that for $d \neq 0$, (1) is not quasi-self-adjoint.

Setting $v=h(t, x, u)$, we study

$$
\left.E^{*}\right|_{v=h(t, x, u)}=\lambda\left(t, x, u, u_{(1)}, \ldots\right) E,
$$

where

$$
\begin{gathered}
v_{t}=D_{t}[h(t, x, u)]=h_{u}(t, x, u) \cdot u_{t}+h_{t}(t, x, u), \\
v_{x}=D_{x}[h(t, x, u)]=h_{u}(t, x, u) \cdot u_{x}+h_{x}(t, x, u), \\
v_{x x}=D_{x}\left(v_{x}\right)=h_{u}(t, x, u) \cdot u_{x x}+h_{u u}(t, x, u) \\
\cdot u_{x}^{2}+2 h_{x u}(t, x, u) \cdot u_{x}+h_{x x}(t, x, u) .
\end{gathered}
$$

The reckoning shows that

$$
\begin{gathered}
\lambda=h_{u}(t, x, u)=0, \\
-h(t, x, u) b(x)+2 h(t, x, u) u \\
-h_{t}(t, x, u)-d h_{x x}(t, x, u)=0 .
\end{gathered}
$$

Equation (59) should be satisfied identically in $t, x$, and $u$. Therefore we nullify the coefficient of $u$ and obtain $h(t, x, u)=$ 0 . According to Definition 6, we claim that for $d \neq 0$, (1) is not nonlinearly self-adjoint.

Hence, for $d \neq 0,(1)$ is neither quasi-self-adjoint nor nonlinearly self-adjoint.

\section{Conservation Laws}

We apply Theorem 2 to (1) together with its adjoint equation (53). With the help of its point symmetries, we can obtain conserved vectors $\left(C^{1}, C^{2}\right)$, which satisfy the conservation equation $\left.\left(D_{t} C^{1}+D_{x} C^{2}\right)\right|_{E=0, E^{*}=0}=0$.

For Case 1 we have $w=-u_{t}$ and (12) yields the conservation law with

$$
\begin{aligned}
& C^{1}=v\left[-d u_{x x}-b(x) u+u^{2}\right], \\
& C^{2}=-d u_{t} v_{x}+d v u_{t x} .
\end{aligned}
$$

This vector involves an arbitrary solution $v$ of the adjoint equation (53) and hence provides an infinite number of conservation laws. With the aid of Mathematica, we find

$$
D_{t} C^{1}+D_{x} C^{2}=u_{t}\left[-d v_{x x}-v_{t}-b(x) v+2 u v\right] .
$$

For Case 2, we find the conservation law provided by $V_{2}$. We have $w=-u-(1 / 2) x u_{x}-t u_{t}$ and (12) yields the nontrivial conservation law with

$$
\begin{aligned}
C^{1} & =v\left(t u^{2}-t \frac{c_{4}}{x^{2}} u-u-\frac{1}{2} x u_{x}-t d u_{x x}\right) \\
C^{2}= & \frac{1}{2} x v\left(u_{t}-\frac{c_{4}}{x^{2}} u+u^{2}\right) \\
& -d v_{x}\left(u+\frac{1}{2} x u_{x}+t u_{t}\right)+d v\left(\frac{3}{2} u_{x}+t u_{t x}\right) .
\end{aligned}
$$

This vector involves an arbitrary solution $v$ of the adjoint equation (53) and hence provides an infinite number of conservation laws. With the aid of Mathematica, we find

$$
\begin{aligned}
D_{t} C^{1} & +D_{x} C^{2} \\
= & \left(u+\frac{1}{2} x u_{x}+t u_{t}\right)\left(-d v_{x x}-v_{t}-\frac{c_{4}}{x^{2}} v+2 u v\right) \\
& -\frac{1}{2} v\left(u_{t}-d u_{x x}-\frac{c_{4}}{x^{2}} u+u^{2}\right) .
\end{aligned}
$$

For Case 3, we find the conservation law provided by the symmetry $V_{3}$. We have $w=-u_{x}$ and (12) yields the nontrivial conservation law with

$$
\begin{aligned}
& C^{1}=-u_{x} v, \\
& C^{2}=v\left(u_{t}-b u+u^{2}\right)-d u_{x} v_{x} .
\end{aligned}
$$

This vector involves an arbitrary solution $v$ of the adjoint equation (53) and hence provides an infinite number of conservation laws. With the aid of Mathematica, we find

$$
D_{t} C^{1}+D_{x} C^{2}=u_{x}\left(-d v_{x x}-v_{t}-b v+2 u v\right) .
$$

According to the definition of conservation law, we say that we have obtained local conservation laws for the simultaneous system of (1) and its adjoint equation (53); however, they are nonlocal for the single equation (1). Then we will illustrate that there is no local conservation law for (1). 
In view of Theorem 8 , for (1) we have the density $T=$ $T(t, x, u)$ and the flux $X=X\left(t, x, u, u_{x}\right)$. We substitute the expression of $u_{t}$ deduced from (1) into

$$
\left.\left(D_{t} T+D_{x} X\right)\right|_{(1)}=0
$$

and we obtain

$$
\begin{aligned}
T_{t}+ & T_{u}\left[d u_{x x}+u(b(x)-u)\right] \\
& +X_{x}+X_{u} u_{x}+X_{u_{x}} u_{x x}=0
\end{aligned}
$$

The coefficient of $u_{x x}$ gives

$$
d T_{u}(t, x, u)+X_{u_{x}}\left(t, x, u, u_{x}\right)=0 ;
$$

therefore

$$
X\left(t, x, u, u_{x}\right)=-d T_{u}(t, x, u) u_{x}+\widehat{X}(t, x, u) .
$$

Taking into account (69) and splitting the rest of (67) with respect to the powers of $u_{x}$, we obtain the system of PDEs on the functions $T(t, x, u)$ and $\widehat{X}(t, x, u)$

$$
\begin{gathered}
u_{x}:-d T_{u x}(t, x, u)+\widehat{X}_{u}(t, x, u)=0 \\
u_{x}^{2}:-d T_{u u}(t, x, u)=0 \\
1: T_{t}(t, x, u)+T_{u}(t, x, u) u(b(x)-u)+\widehat{X}_{x}(t, x, u)=0 .
\end{gathered}
$$

For $d \neq 0$, according to (71), we can assume

$$
T(t, x, u)=\phi(t, x) u+T^{0}(t, x) ;
$$

then

$$
\widehat{X}_{u}(t, x, u)=d \phi_{x}(t, x),
$$

so we assume

$$
\widehat{X}(t, x, u)=d \phi_{x}(t, x) u+X^{0}(t, x) .
$$

Now

$$
X\left(t, x, u, u_{x}\right)=-d \phi(t, x) u_{x}+d \phi_{x}(t, x) u+X^{0}(t, x) .
$$

Substitution of $T(t, x, u)$ and $X\left(t, x, u, u_{x}\right)$ obtained into (72) yields

$$
\begin{gathered}
\phi_{t}(t, x) u+T_{t}^{0}(t, x)+\phi(t, x) u(b(x)-u) \\
+d \phi_{x x}(t, x) u+X_{x}^{0}(t, x)=0
\end{gathered}
$$

We can set $T^{0}(t, x)=X^{0}(t, x)=0$ as they only contribute to the trivial part of the conservation law. Taking into account the remainder of the later expression and splitting it with respect to the powers of $u$, we obtain the system of PDEs on $\phi(t, x)$

$$
\begin{gathered}
u: \phi_{t}(t, x)+d \phi_{x x}(t, x)+b(x) \phi(t, x)=0, \\
u^{2}:-\phi(t, x)=0 .
\end{gathered}
$$

It is clear that the only solution of this system is $\phi(t, x)=0$. It means that (1) does not have any local conservation law.

\section{Some Exact Solutions}

In this section, we will construct scale-invariant solution for (48) and traveling wave solutions for (51).

5.1. Scale-Invariant Solution of (48). For (48), we consider

$$
V_{2}=\frac{1}{2} x \partial_{x}+t \partial_{t}-u \partial_{u}
$$

which corresponds to the scaling group

$$
(x, t, u) \longmapsto\left(\lambda^{1 / 2} x, \lambda t, \lambda^{-1} u\right), \quad \lambda \in \mathbb{R}^{+} .
$$

On the half space $\{(x, t, u), t>0\}$, global invariants of this one-parameter group are provided by the functions

$$
y=t^{-(1 / 2)} x, \quad v=t u
$$

then

$$
\begin{aligned}
u & =t^{-1} v, \\
u_{x} & =t^{-1} v_{y}, \\
u_{x x} & =t^{-2} v_{y y}, \\
u_{t} & =-t^{-2}\left(v+\frac{1}{2} y v_{y}\right) .
\end{aligned}
$$

Substitutions into (48) yield

$$
-t^{-2}\left(v+\frac{1}{2} y v_{y}\right)=t^{-2}\left(d v_{y y}+c_{4} y^{-2} v+v^{2}\right) .
$$

As guaranteed by the general theory, this equation is equivalent to one in which the parametric variable $t$ does not occur; namely,

$$
d v_{y y}+\frac{1}{2} y v_{y}+v+c_{4} y^{-2} v-v^{2}=0
$$

which forms the reduced equation for the scale-invariant solutions.

If we get a solution of this equation, we can construct a scale-invariant solution of (48).

When we used the odeadvisor command of Maple for classifying (84) according to standard text books, it returns to $[\mathrm{NONE}]$. The usual substitution

$$
v^{\prime}(y)=\phi(v)
$$

reduces $(84)$ to the first-order equation

$$
d \phi(v) \phi^{\prime}(v)+\frac{1}{2} y \phi(v)+v+c_{4} y^{-2} v-v^{2}=0 .
$$

For (86) the odeadvisor command returns to [rational, [Abel, 2nd type, class A]]; that is, (86) is a class A of second kind of Abel's equation, whose general form is

$$
(y(x)+g(x)) y^{\prime}(x)=f_{2}(x) y(x)^{2}+f_{1}(x) y(x),
$$

where $f_{2}(x), f_{1}(x)$ are arbitrary functions. According to [26], there is as yet no general solution for this ordinary differential equation. 
5.2. Traveling Wave Solutions of (51). Noting that (51), when $d=1$, is a special case of

$$
\frac{\partial w}{\partial t}=\frac{\partial^{2} w}{\partial x^{2}}+a w+b_{1} w^{m}
$$

with $a=b, b_{1}=1, m=2$. So according to the formulae in [27], one can obtain the traveling wave solutions

$$
\begin{gathered}
u(x, t)=[\beta+C \exp (\lambda t \pm \mu x)]^{-2}, \\
u(x, t)=[-\beta+C \exp (\lambda t \pm \mu x)]^{-2},
\end{gathered}
$$

where $\lambda=-(5 / 6) b, \mu=\sqrt{b / 6}$, and $\beta=\sqrt{1 / b}$.

Remark 9. The solutions (89) have been verified by the pdetest command of Maple.

If $d \neq 1$, for (51), we consider the translation group

$$
(x, t, u) \longmapsto(x+c \epsilon, t+\epsilon, u), \quad \epsilon \in \mathbb{R},
$$

generated by $\partial_{t}+c \partial_{x}$, in which $c$ is a fixed constant and determines the speed of the waves. Global invariants of this group are

$$
y=x-c t, \quad v=u,
$$

so that a group-invariant solution $v=h(y)$ takes the familiar form $u=h(x-c t)$ determining a wave of unchanging profile moving at the constant velocity $c$. Solving for the derivatives of $u$ with respect to $x$ and $t$ in terms of those of $v$ with respect to $y$ we find

$$
u_{t}=-c v_{y}, \quad u_{x}=v_{y}, \quad u_{x x}=v_{y y} .
$$

Substituting these expressions into (51), we find the reduced ordinary differential equation for the traveling wave solution to be

$$
d v_{y y}+c v_{y}+b v-v^{2}=0
$$

This is a nonlinear, constant coefficient equation. If we get a solution of this equation, we can construct a traveling wave solution of (51).

This is clearly invariant that under the group of translations in the $y$-direction

$$
(y, v) \longmapsto(y+\epsilon, v),
$$

with infinitional generator $V=\partial_{y}$. We can construct canonical variables

$$
\tau=\tau(y, v), \quad w=w(y, v),
$$

by solving the equations

$$
\frac{\partial \tau}{\partial y}=0, \quad \frac{\partial w}{\partial y}=1
$$

and we obtain

$$
\tau(y, v)=v, \quad w(y, v)=y .
$$

Then

$$
\frac{d v}{d y}=\frac{1}{w_{\tau}}, \quad \frac{d^{2} v}{d y^{2}}=-\frac{w_{\tau \tau}}{w_{\tau}^{3}},
$$

so (93) becomes

$$
d\left(-\frac{w_{\tau \tau}}{w_{\tau}^{3}}\right)+c \frac{1}{w_{\tau}}+b \tau-\tau^{2}=0,
$$

which is a first-order equation for $z=w_{\tau}$ :

$$
d\left(-\frac{z_{\tau}}{z^{3}}\right)+c \frac{1}{z}+b \tau-\tau^{2}=0
$$

that is

$$
d z_{\tau}=\left(b \tau-\tau^{2}\right) z^{3}+c z^{2}
$$

Setting $z=1 / \omega$, we obtain

$$
\omega \frac{d \omega}{d \tau}=-\frac{1}{d}\left(b \tau-\tau^{2}\right)-\frac{c}{d} \omega
$$

With the aid of the substitution $\xi=-(c / d) \tau$, (102) becomes

$$
\omega \frac{d \omega}{d \xi}=\omega+\left(-\frac{b d}{c^{2}} \xi-\frac{d^{2}}{c^{3}} \xi^{2}\right) .
$$

When $b d / c^{2}= \pm 6 / 25$, according to [28], one can get the solution of (103) by the elliptic Weierstrase function. That is, if $b d / c^{2}=6 / 25$, the solution of (103) in the parametric form is

$$
\xi=5 a r^{2} \varphi, \quad \omega=a r^{2} E_{1},
$$

where $a=-\left(125 d^{2} / 6 c^{3}\right), \quad r=\int\left(d \varphi / \pm \sqrt{4 \varphi^{3}-1}\right)-c_{2}, \varphi$ is the classical elliptic Weierstrase function $\varphi=\varphi\left(r+c_{2}, 0,1\right)$, and $E=r \sqrt{ \pm\left(4 \varphi^{3}-1\right)}+2 \varphi$, where $c_{2}$ is a constant; if $b d / c^{2}=$ $-6 / 25$, the solution in the parametric form is

$$
\xi=5 a E_{2}, \quad \omega=a r^{2} E_{1},
$$

with $E_{2}=r^{2} \varphi \mp 1$.

\section{Concluding Remarks}

Some exact solutions and a discussion on local conservation laws for the variable-coefficient reaction-diffusion equation were presented. Firstly, by solving the determining equations in Lie symmetry analysis, we classified the equation into three kinds. For every kind, we obtained the Lie symmetries. Corresponding to these Lie point symmetries, we have expressed the conservation laws, respectively. For (48), we studied its scale-invariant solution by global invariants of one-parameter group $V_{2}$. Each solution of (84) leads to a scale-invariant solution of (48). For (51), we have obtained some exact solutions. 


\section{Conflict of Interests}

The authors declare that there is no conflict of interests regarding the publication of this paper.

\section{Acknowledgments}

The authors are grateful to the referee for his/her valuable comments and suggestions which have led to an improvement of the presentation. This research is supported by the National Natural Science Foundation of China (no. 11061016).

\section{References}

[1] E. Noether, "Invariante variationsprobleme, königliche gesellschaft der wissenschaften zu Göttingen, Nachtichten," Mathematisch-Physikalische Klasse Heft, vol. 2, pp. 235-257, 1918.

[2] P. J. Olver, Applications of Lie Groups to Differential Equations, vol. 107, Springer, New York, NY, USA, 1986.

[3] N. H. Ibragimov, "A new conservation theorem," Journal of Mathematical Analysis and Applications, vol. 333, no. 1, pp. 311328,2007

[4] A. H. Kara and F. M. Mahomed, "Relationship between symmetries and conservation laws," International Journal of Theoretical Physics, vol. 39, no. 1, pp. 23-40, 2000.

[5] A. H. Kara and F. M. Mahomed, "Noether-type symmetries and conservation laws via partial Lagrangians," Nonlinear Dynamics, vol. 45, no. 3-4, pp. 367-383, 2006.

[6] S. C. Anco and G. Bluman, "Direct construction of conservation laws from field equations," Physical Review Letters, vol. 78, no. 15, pp. 2869-2873, 1997.

[7] G. W. Bluman and S. C. Anco, Symmetry and Integration Methods for Differential Equations, vol. 154 of Applied Mathematical Sciences, Springer, New York, NY, USA, 2002.

[8] G. W. Bluman, Temuerchaolu, and S. C. Anco, "New conservation laws obtained directly from symmetry action on a known conservation law," Journal of Mathematical Analysis and Applications, vol. 322, no. 1, pp. 233-250, 2006.

[9] N. H. Ibragimov, "Integrating factors, adjoint equations and Lagrangians," Journal of Mathematical Analysis and Applications, vol. 318, no. 2, pp. 742-757, 2006.

[10] B. Muatjetjeja and C. M. Khalique, "First integrals for a generalized coupled Lane-Emden system," Nonlinear Analysis: Real World Applications, vol. 12, no. 2, pp. 1202-1212, 2011.

[11] H. Steudel, "Noether's theorem and the conservation laws of the Korteweg-de Vries equation," Annalen der Physik, vol. 32, no. 6, pp. 445-455, 1975.

[12] N. H. Ibragimov, "Nonlinear self-adjointness and conservation laws," Journal of Physics A: Mathematical and Theoretical, vol. 44, no. 43, Article ID 432002, 2011.

[13] N. H. Ibragimov, "Nonlinear self-adjointness in constructing conservation laws," Arch Mikrobiol, vol. 7-8, pp. 1-90, 2011.

[14] I. L. Freire, "Conservation laws for self-adjoint first-order evolution equation," Journal of Nonlinear Mathematical Physics, vol. 18, no. 2, pp. 279-290, 2011.

[15] I. L. Freire, "New conservation laws for inviscid Burgers equation," Computational and Applied Mathematics, vol. 31, no. 3, pp. 559-567, 2012.
[16] N. H. Ibragimov, R. S. Khamitova, and A. Valenti, "Self-adjointness of a generalized Camassa-Holm equation," Applied Mathematics and Computation, vol. 218, no. 6, pp. 2579-2583, 2011.

[17] R. Naz, F. M. Mahomed, and D. P. Mason, "Comparison of different approaches to conservation laws for some partial differential equations in fluid mechanics," Applied Mathematics and Computation, vol. 205, no. 1, pp. 212-230, 2008.

[18] R. Morris and A. H. Kara, "New conservation laws of some third-order systems of pdes arising from higher-order multipliers," Applied Mathematics and Computation, vol. 217, no. 6, pp. 2639-2643, 2010.

[19] M. L. Gandarias and M. S. Bruzon, "Conservation laws for a class of quasi self-adjoint third order equations," Applied Mathematics and Computation, vol. 219, no. 2, pp. 668-678, 2012.

[20] R. S. Cantrell and C. Cosner, "Diffusive logistic equations with indefinite weights: population models in disrupted environments," Proceedings of the Royal Society of Edinburgh A Mathematics, vol. 112, no. 3-4, pp. 293-318, 1989.

[21] P. A. Clarkson and E. L. Mansfield, "Symmetry reductions and exact solutions of a class of nonlinear heat equations," Physica D. Nonlinear Phenomena, vol. 70, no. 3, pp. 250-288, 1994.

[22] T. Ide and M. Okada, "Numerical simulation for a nonlinear partial differential equation with variable coefficients by means of the discrete variational derivative method," Journal of Computational and Applied Mathematics, vol. 194, no. 2, pp. 425-459, 2006.

[23] R. Popovych, "Direct methods of construction of conservation laws," Physics AUC II, vol. 16, pp. 81-94, 2006.

[24] N. H. Ibragimov, "Quasi-self-adjoint differential equations," Archives of ALGA, vol. 4, pp. 55-60, 2007.

[25] M. L. Gandarias, "Weak self-adjoint differential equations," Journal of Physics A: Mathematical and Theoretical, vol. 44, no. 26, Article ID 262001, 2011.

[26] E. Kamke, Differentialgleichungen : Lösungsmethoden und Lösuneg, vol. 2, American Mathematical Society, 1974.

[27] A. D. Polyanin and F. V. Zaitsev, Handbook of Nonlinear Partial Differential Equations, CRC press, New York, NY, USA, 2004.

[28] V. F. Zaitsev and A. D. Polyanin, Handbook of Exact Solutions for Ordinary Differential Equations, CRC Press, 2002. 


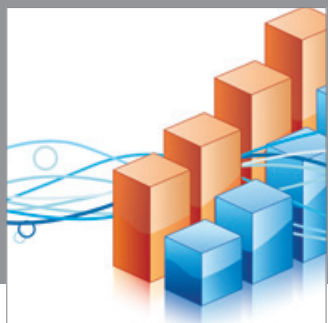

Advances in

Operations Research

mansans

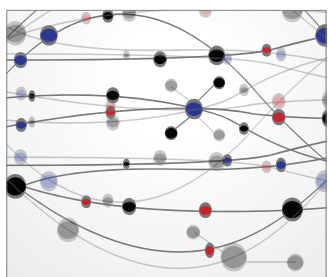

The Scientific World Journal
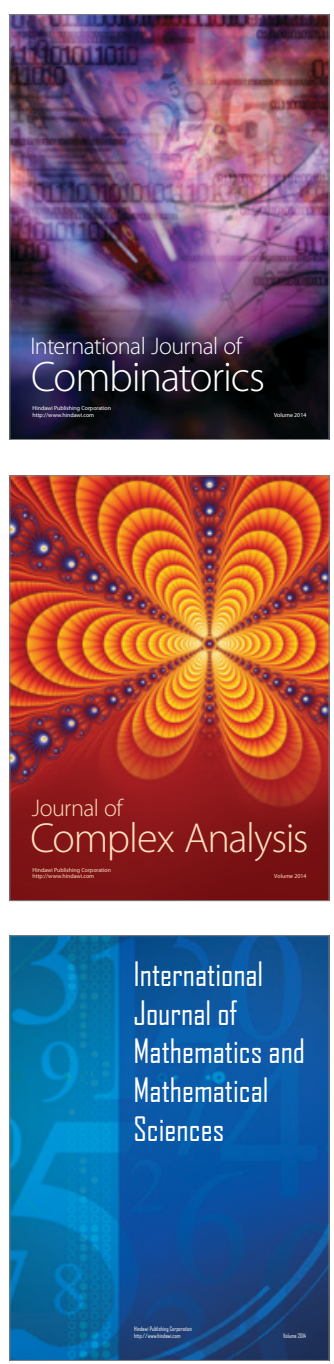
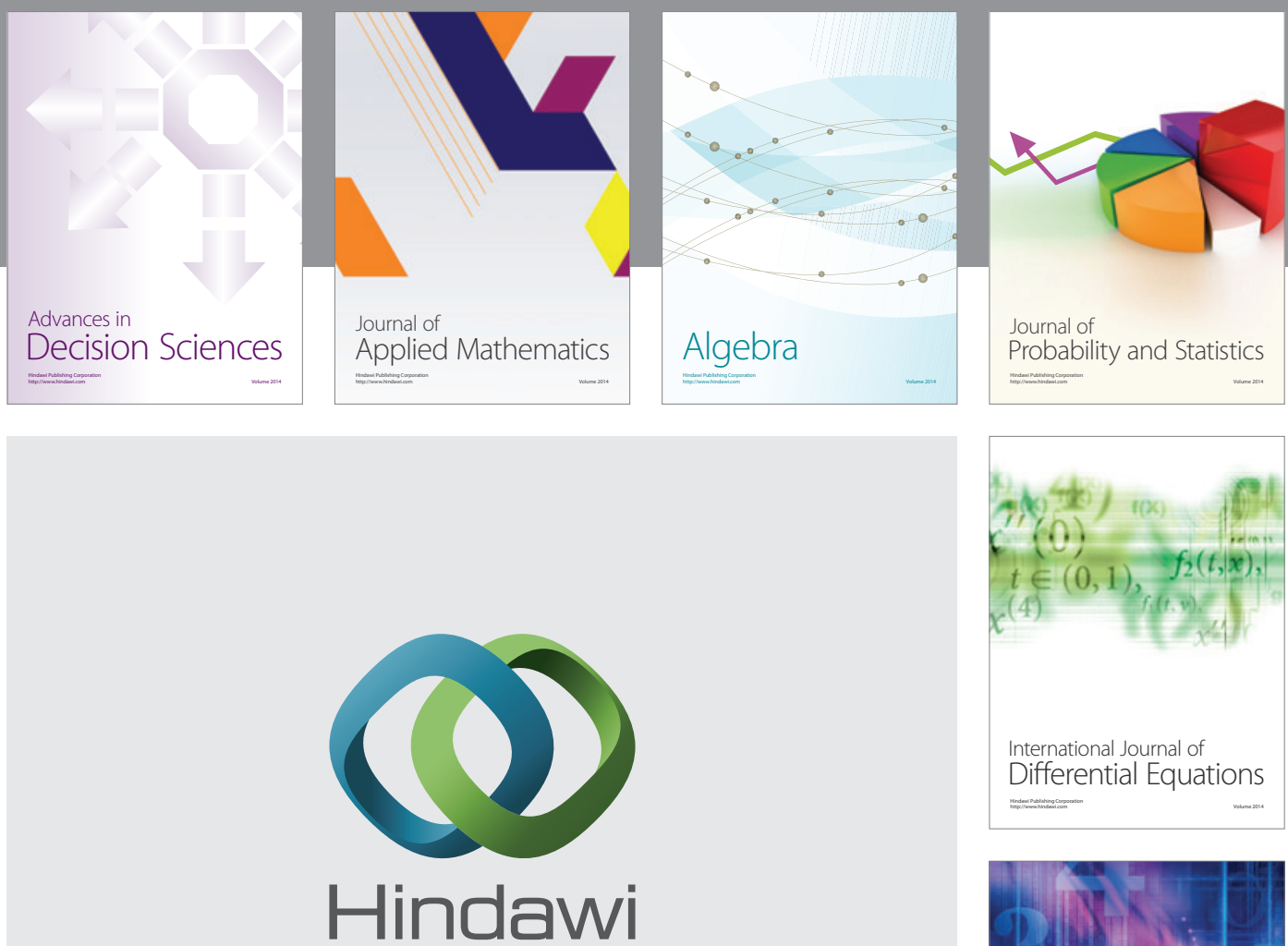

Submit your manuscripts at http://www.hindawi.com
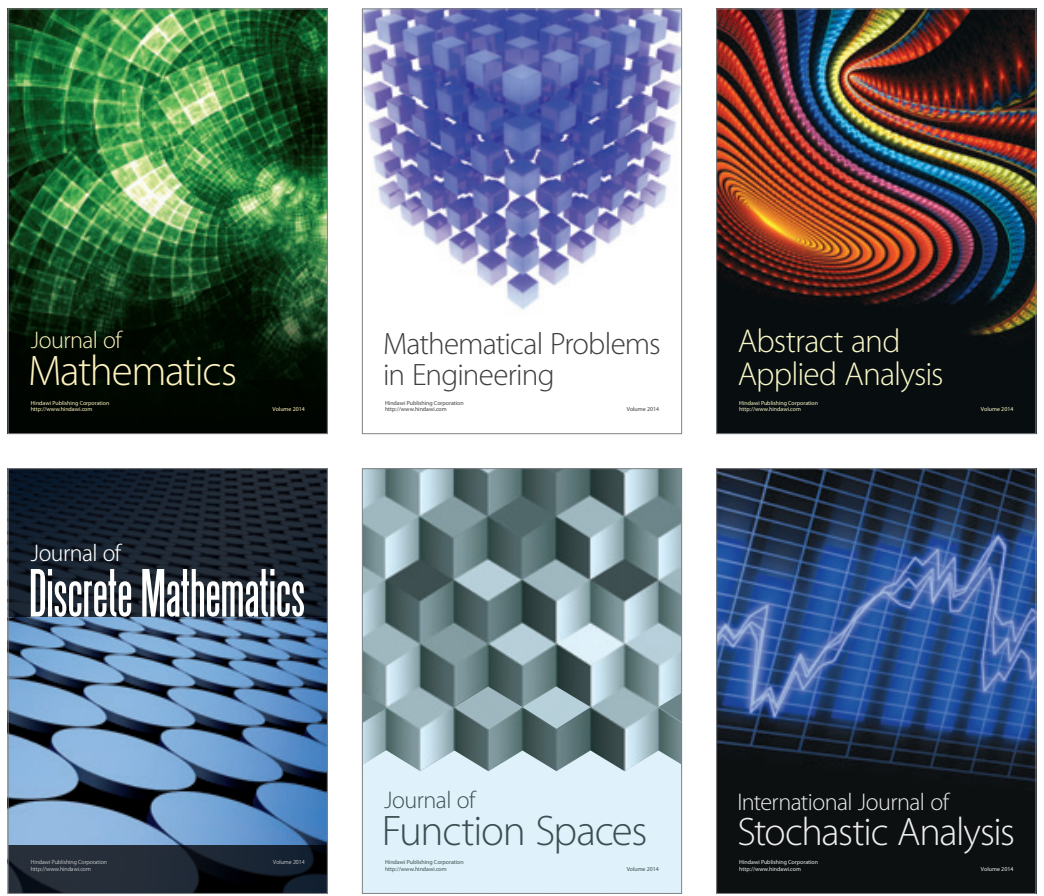

Journal of

Function Spaces

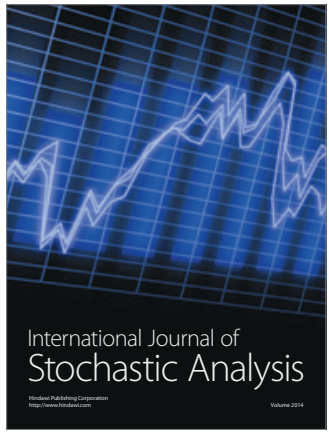

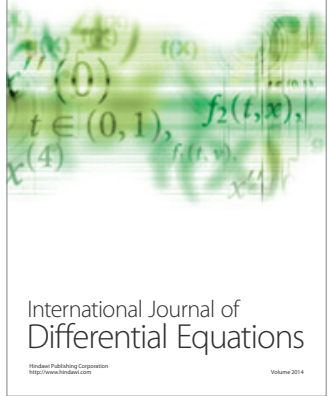
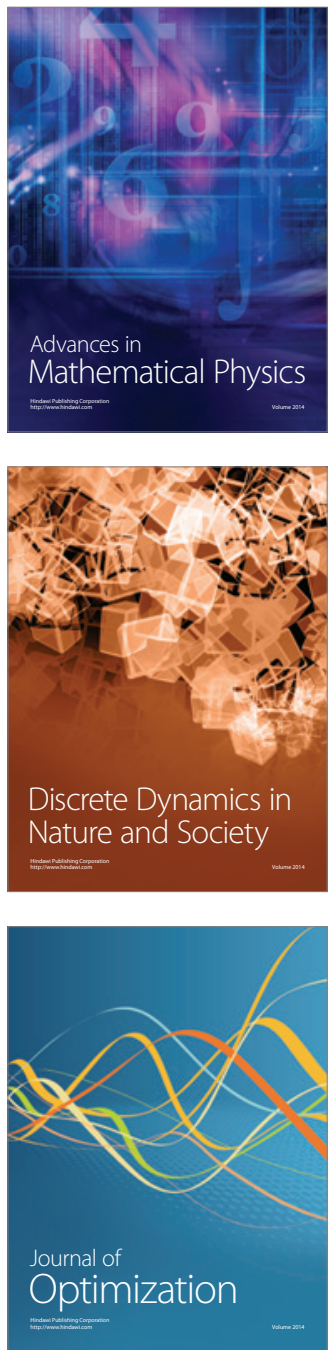